\title{
GEOLOGICAL SURVEY OF JAPAN RADIOCARBON DATES I
}

\author{
SHIGEKO TOGASHI and EIJI MATSUMOTO \\ Geological Survey of Japan, Tsukuba, Ibaraki 305, Japan
}

This radiocarbon laboratory began operation in 1980 using the benzene scintillation method. The benzene synthesizer is essentially identical with that of Ikeda (1976). A liquid scintillation counter is Aloca LSC-LB1. Samples dated are wood, charcoal, shell, and coral.

Pretreatment of wood and charcoal is a standard acid-alkali procedure, using $2 \% \mathrm{HCl}$ and $2 \% \mathrm{NaOH}$ at elevated temperatures. Charcoal is further heated in concentrated $\mathrm{HNO}_{3}$ for one hour, diluted in water, stands one night, and is washed and dried. Pretreated wood and charcoal are carbonized before combustion. The combustion products are passed over $\mathrm{CuO}$, and are collected in an ammonium hydroxide bubbler system, and precipitated with calcium chloride.

Carbonate samples such as shell and coral are washed in diluted $\mathrm{HCl}$ and, subsequently, organic matter in carbonate is carbonized before conversion to $\mathrm{CO}_{2}$.

Standard oxalic acid is oxidized by the wet method of Valastro, Land, and Varela (1977). $\mathrm{CO}_{2}$ is converted to benzene through lithium carbide and acetylene basically by the methods of Noakes, Kim, and Stipp (1965) and Kim, Ikeda, and Ruch (1969). The catalyst used to synthesize benzene can be easily made in the laboratory. The silica alumina catalyst base is activated by boiled ammonium metavanadate solution, allowed to stand one night, and is washed and dried.

Memory effect in the stainless steel reaction vessel can be removed by using an exchangeable inner vessel and by baking it in the air.

Counting efficiency is $65 \%$ and background rate is $0.9 \mathrm{cpm}$ for a mixture of $4 \mathrm{ml}$ benzene and $2 \mathrm{ml}$ scintillator in a low potassium glass vial and $1.8 \mathrm{cpm}$ for a mixture of $15 \mathrm{ml}$ benzene and $5 \mathrm{ml}$ scintillator in a teflon vial. Each sample including background and sealed reference standard is placed in the counter and counted sequentially for $50 \mathrm{~min}$. The cycle is repeated as often as desired with a minimum of 20 cycles, $1000 \mathrm{~min} / \mathrm{sam}$ ple, for each series of determinations.

Quenching is corrected, especially for young samples, because it varies the counting efficiency range of $\pm 1.5 \%$.

Dates are calculated based on 0.95 of the activity of NBS oxalic acid (SRM-4990) and the Libby half-life for ${ }^{14} \mathrm{C}$ of $5570 \pm 30$ years. Errors quoted are $2 \sigma$ statistical error. The maximum measurable age under routine condition of $3 \mathrm{ml}$ benzene sample and 1000 minute counting is 40,000 years.

The activity of the NBS standard (SRM 4990) is measured to be 13.99 $\pm 0.30(2 \sigma) \mathrm{dpm} / \mathrm{gC}$ and that of the New NBS standard (RM 49) is measured to be $18.34 \pm 0.25(2 \sigma) \mathrm{dpm} / \mathrm{gC}$. Paleozoic limestone is measured for a blank test to be $>39,500$ years BP under the routine condition of $2.2 \mathrm{~g}$ carbon and 1000 minute counting. 
Samples previously measured in other laboratories were dated in our laboratory. The results of this cross checking are given in table 1 which shows that obtained dates are in good agreement with reported dates.

\section{ACKNOWLEDGMENTS}

Thanks are due S Ikeda, Tokyo Technical University, and H Shirakawa, Tsukuba University, for their kind advice. We would like to thank K Kigoshi and S Suzuki, Gakushuin University, for helpful suggestions and samples for cross checking. We wish also to express our thanks to $\mathrm{Y}$ Maeda, Kobe Educational Institute, Y Hayakawa, Tokyo University, and $\mathrm{N}$ Isshiki and $\mathrm{H}$ Yamazaki, Geological Survey of Japan, for providing the samples for cross checking.

\section{SAMPLE DESGRIPTIONS}

\section{GEOLOGIC SAMPLES}

\section{Asama Volcano series}

$$
\text { Japan }
$$

Charcoal from tree in First Pumice Flow deposit of Asama Volcano, central Japan. Coll 1981 by S Togashi.
JGS-16. Asama
$\mathbf{1 3 , 5 0 0} \pm \mathbf{5 0 0}$

Charcoal from river cut at Manza-kazawaguchi, Gumma (36 $31^{\prime} 40^{\prime \prime}$ $\left.\mathrm{N}, 138^{\circ} 31^{\prime} 15^{\prime \prime} \mathrm{E}\right), 30 \mathrm{~m}$ below surface.

\section{JGS-36. Asama}

$13,700 \pm 400$

Same sample as JGS-16.

JGS-37. Asama

$13,600 \pm 400$

Charcoal from Komoro, Nagano (36 $\left.26^{\circ} 05^{\prime \prime} \mathrm{N}, 138^{\circ} 27^{\prime} 15^{\prime \prime} \mathrm{E}\right), 4 \mathrm{~m}$ below surface.

TABLE 1

Cross-check samples

\begin{tabular}{|c|c|c|c|c|c|c|c|}
\hline $\begin{array}{l}\text { Sample } \\
\text { no. }\end{array}$ & & JGS no. & JGS date & $\begin{array}{l}\text { Other } \\
\text { lab no. }\end{array}$ & Other dates & $\begin{array}{c}\text { Difference } \\
\text { between dates }\end{array}$ & Submitter \\
\hline $1 *$ & Wood & JGS-24 & $940 \pm 200$ & GaK- 9229 & $950 \pm 110$ & 10 & Yamazaki \\
\hline 2 & Charcoal & -48 & $1170 \pm 170$ & -10046 & $1090 \pm 100$ & 80 & Hayakawa \\
\hline 3 & Wood & -20 & $2360 \pm 170$ & - 9312 & $2430 \pm 110$ & 70 & Kigoshi \\
\hline $3^{\prime}$ & Wood & -26 & $2470 \pm 170$ & - 9312 & $2430 \pm 110$ & 40 & Kigoshi \\
\hline 4 & Wood & -49 & $13,040 \pm 410$ & -10043 & $12,460 \pm 520$ & 580 & Hayakawa \\
\hline 5 & Wood & -57 & $21,350 \pm 710$ & -8745 & $21,810 \pm 150$ & 460 & Kigoshi \\
\hline 6 & Charcoal & -89 & $\begin{array}{r}34,000+2500 \\
-1900\end{array}$ & -1589 & $\begin{array}{r}30,900+700 \\
-700\end{array}$ & 3100 & Isshiki \\
\hline $7 * *$ & Charcoal & JGS-50 & $>37,200$ & GaK-10048 & $>28,070$ & - & Hayakawa \\
\hline 8 & Shell & -61 & $5800 \pm 220$ & N-2942 & $5800 \pm 110$ & 0 & Maeda \\
\hline 9 & Shell & -59 & $6040 \pm 220$ & N-1305 & $5960 \pm 120$ & 80 & Maeda \\
\hline 10 & Shell & -60 & $6990 \pm 220$ & GaK- 3757 & $6600 \pm 150$ & $390+$ & Maeda \\
\hline 11 & Shell & -11 & $7300 \pm 220$ & N-3085 & $7330 \pm 120$ & 30 & Maeda \\
\hline
\end{tabular}

* Samples 1-6 are the same as GaK samples under "Other lab no."

** Samples 7-11 are different but from the same formations and outcrops as those under "Other lab no."

+ The large difference in the dates of sample 10 may be attributed to the difference in samples. However, both dates are consistent with the stratigraphy. 
JGS-40. Asama

Same sample as JGS-37.

\section{O-shima Volcano series}

Wood in volcaniclastic flow deposit of older edifice of pre-caldera stratovolcano of O-shima Volcano, Izu Is. Coll 1983 by N Isshiki and H Matsuura, Geol Survey Japan.

JGS-99. O-shima, NI83042401 $\left.13^{\prime \prime} \mathrm{E}\right)$.

Wood from sea cliff W of Okata, O-shima $\left(34^{\circ} 47^{\prime} 11^{\prime \prime} \mathrm{N}, 139^{\circ} 23^{\prime}\right.$

\section{JGS-100. O-shima, NI83042402}

Wood from same deposit as JGS-99.

\section{Hachijo-jima Volcano}

Charcoal in pumice flow deposit of pre-caldera stratovolcano of Higashi-yama Volcano in Hachijo-jima, Izu Is. Coll 1967 by N Isshiki.

JGS-89. Hachijo, NI67091703

Charcoal from near N mouth of Osaka Tunnel, S of Osato, Hachijomachi $\left(33^{\circ} 05^{\prime} 50^{\prime \prime} \mathrm{N}, 139^{\circ} 47^{\prime} 15^{\prime \prime} \mathrm{E}\right)$.

\section{Miyake-jima Volcano}

Charcoal in spatter deposit formed along fissure system, which was generated just before caldera formation of Miyake-jima Volcano, Izu Is. Coll 1982 by N Isshiki.

JGS-46. Miyake-jima, NI82052702

$3030 \pm 310$ $\left.29^{\prime} 35^{\prime \prime}\right)$.

Charcoal from W flank of Miyake-jima Volcano $\left(34^{\circ} 05^{\prime} 10^{\prime \prime} \mathrm{N}, 139^{\circ}\right.$

JGS-85. Miyake-jima, NI82052702

$2880 \pm 180$

Same sample as JGS-46.

\section{Sakura River deposit series}

Wood from deposit by Sakura R, Tsukuba, Ibaraki. Coll 1980 and 1981 by $\mathrm{S}$ Togashi, and $\mathrm{H}$ Ikeda and F Iseya, Tsukuba Univ. Sample measured to date route change of Sakura R.

JGS-27.

$2460 \pm 180$

Wood from Yasumori, Tsukuba $\left(36^{\circ} 11^{\prime} 0^{\prime \prime} \mathrm{N}, 140^{\circ} 04^{\prime} 25^{\prime \prime} \mathrm{E}\right), 2 \mathrm{~m}$ below surface, middle part of deposit of Sakura R.

JGS-72.

$21,740 \pm 730$

Wood from Tanaka, Tsukuba $\left(36^{\circ} 10^{\prime} 25^{\prime \prime} \mathrm{N}, 140^{\circ} 04^{\prime} 30^{\prime \prime} \mathrm{E}\right), 2 \mathrm{~m}$ below surface, bottom of deposit of Sakura R. 


\section{Pacific Ocean}

\section{Tinian Island, southern Mariana Islands series}

Coral from E coast of Tinian I. $\left(15^{\circ} 00^{\prime} \mathrm{N}, 145^{\circ} 40^{\prime} \mathrm{E}\right)$. Coll 1982 by H Kayane, N Yonekura, and Y Ida, Tokyo Univ. Samples measured to date sea-level change along $S$ Mariana Is. coast.

\section{JGS-75. TINIAN-3.82}

Coral from $2.9 \mathrm{~m}$ above msl. Sample is aragonite.

JGS-76. TINIAN-5.82

Coral from $2.2 \mathrm{~m}$ above msl. Sample is aragonite.

\section{Rota Island, southern Mariana Islands series}

Coral from W coast of Rota I. (14. $\left.03^{\prime} \mathrm{N}, 145^{\circ} 10^{\prime} \mathrm{E}\right)$. Coll 1982 by $\mathrm{H}$ Kayane, N Yonekura, and Y Ida. Samples measured to date sea-level change along $S$ Mariana Is. coast.

JGS-77. ROTA D-1.82

$16,500 \pm 400$ calcite.

Coral from $5 \mathrm{~m}$ above msl. Sample is aragonite, including $3 \% \mathrm{Mg}-$

JGS-78. ROTA D-2.82

$4070 \pm 190$ calcite.

Coral from $3.3 \mathrm{~m}$ above msl. Sample is aragonite, including $1 \% \mathrm{Mg}-$

\section{JGS-79. ROTA D-3.82}

Coral from $4.7 \mathrm{~m}$ above msl. Sample is aragonite, including $1 \%$ calcite.

\section{JGS-80. ROTA D-4.82}

$4040 \pm 190$

Coral from $3.1 \mathrm{~m}$ above msl. Sample is $99 \%$ aragonite.

JGS-81. ROTA D-5.82

$3780 \pm 200$

Coral from $1.6 \mathrm{~m}$ above msl. Sample is aragonite.

\section{JGS-82. ROTA D-6.82}

$5010 \pm 200$

Coral from $1.2 \mathrm{~m}$ above msl. Sample is aragonite.

\section{Mangaia Island, southern Cook Islands series}

Coral from drill hole at $\mathrm{W}$ coast of Mangaia I. $\left(22^{\circ} 00^{\prime} \mathrm{S}, 157^{\circ} 40^{\prime}\right.$ W). Height of drilled coral surface is recent sea level. Coll 1982 by N Yonekura and Y Matsushima, Kanagawa Pref Mus, and Y Maeda, Kobe Educ Inst. Samples measured to date sea-level change along Cook Is.

JGS-63. MANGAIA 20.21

$2290 \pm 160$

Coral from $0.8 \mathrm{~m}$ below msl. 
JGS-64. MANGAIA 37

Coral from $1.8 \mathrm{~m}$ below msl.

JGS-65. MANGAIA 63.64

Coral from $3.8 \mathrm{~m}$ below msl. Sample is aragonite, including $6 \%$ calcite.

Mangaia Island, southern Cook Islands series

Coral from coast at NW coast of Mangaia I. (22 $\left.00^{\prime} \mathrm{S}, 157^{\circ} 40^{\prime} \mathrm{W}\right)$. Coll 1982 by N Yonekura, Y Matsushima, and Y Maeda. Samples measured to date sea-level change along Cook Is.

JGS-62. MANGAIA 4.82

$5020 \pm 190$ calcite.

Coral from 1.1 to $1.3 \mathrm{~m}$ above msl. Sample is aragonite, including $1 \%$

JGS-96. MAN 3.82

$\mathbf{3 4 1 0} \pm \mathbf{1 7 0}$

Coral from $1.8 \mathrm{~m}$ above msl.

JGS-97. MAN 1.82

$40,800+8200$

Coral from 1.8 to $2.3 \mathrm{~m}$ above $\mathrm{msl}$.

$-4000$

Aitutaki Island, southern Cook Islands series

Coral from drill hole at $\mathrm{N}$ coast of Aitutaki I. $\left(18^{\circ} 40^{\prime} \mathrm{S}, 160^{\circ} 02^{\prime} \mathrm{W}\right)$. Height of drilled coral surface is recent sea level. Coll 1982 by $\mathrm{N}$ Yonekura, Y Matsushima, and Y Maeda. Samples measured to date sea-level change along Cook Is.

JGS-66. AUTITAKI-16

$4690 \pm 190$

Coral from $0.7 \mathrm{~m}$ below msl.

JGS-67. AUTITAKI-48.49

$5320 \pm 190$

Coral from $2 \mathrm{~m}$ below msl.

Rarotonga Island, southern Cook Islands series

Coral from drill hole at $\mathrm{N}$ coast of Rarotonga I. $\left(21^{\circ} 00^{\prime} \mathrm{S}, 160^{\circ} 00^{\prime}\right.$ $W)$. Height of drilled coral surface is recent sea level. Coll 1982 by N Yonekura, Y Matsushima, and Y Maeda. Samples measured to date sea-level change along Cook Is.

JGS-73. RAROTONGA 24

$6010 \pm 200$

Coral from $1.4 \mathrm{~m}$ below msl.

JGS-74. RAROTONGA 36.37

$6090 \pm 200$

Coral from $1.9 \mathrm{~m}$ below msl.

Viti Levu, Fiji Islands series

Shell and wood from drill holes at S coast of Viti Levu, Fiji. Coll 1982 by A Sugimura, Kobe Univ, Y Matsushima, E Matsumoto, Y Maeda, K Berryman, New Zealand Geol Survey, and T Ishii and N Yonekura, Tokyo Univ. 
JGS-51. FIJI St I

$2640 \pm 330$ $\left.20^{\prime} \mathrm{E}\right)$.

Shell from 2.25 to $2.5 \mathrm{~m}$ below msl at drill hole St $\mathrm{I}\left(18^{\circ} 10^{\prime} \mathrm{S}, 178^{\circ}\right.$

JGS-55. FIJI St III

$4400 \pm 200$ $\left.20^{\prime} \mathrm{E}\right)$.

Shell from 1 to $1.25 \mathrm{~m}$ below msl at drill hole St III $\left(18^{\circ} 10^{\prime} \mathrm{S}, 178^{\circ}\right.$

JGS-98. FIJI St III

$1630 \pm 250$

Wood from $1.75 \mathrm{~m}$ above msl at drill hole St III ( $\left.18^{\circ} 10^{\prime} \mathrm{S}, 178^{\circ} 20^{\prime} \mathrm{E}\right)$.

\section{REFERENCES}

Ikeda, S, 1976, Measurement of weak radiocarbon by benzene liquid scintillation method: Isotope News, v 336, p 6-7.

Kim, S M, Ikeda, S, and Ruch, R R, 1969, Labeling of benzene with carbon and hydrogen isotopes: Radiochem Radioanal Letters, v l, p 379-382.

Noakes, J E, Kim, S M, and Stipp, J J, 1965, Chemical and counting advances in liquid scintillation age counting, in Chatters, $\mathrm{R} M$ and Olson, $\mathrm{E} \mathrm{A}$, eds, Internatl conf on radiocarbon and tritium dating, 6th Proc: Washington, DC, Clearinghouse for fed sci and tech inf, Natl Bur Standards, p 68-92.

Valastro, S, Jr, Land, L S, and Varela, A G, 1977, An improved procedure for wet oxidation of the ${ }^{14} \mathrm{C}$ NBS oxalic acid standard: Radiocarbon, v 19, p 375-382. 